\title{
Performance Comparison of HBTs and Quasi E-mode PHEMTs for Single Supply High Efficiency Power Amplifiers
}

\author{
Y. Tkachenko, C. Wei, S. Sprinkle, J. Gering, J. Lee, T. Kao, Y. Zhao W. Ho, M. Sun and D. Bartle \\ Alpha Industries. Inc., 20 Sylvan Rd, Woburn, MA 01801 and 1230 Bordeaux Dr, Sunnyvale, CA 94089
}

Tel: 781-935-5150; E-mail: gtkachenko@alphaind.com

\begin{abstract}
Performance of an HBT and a Quasi Enhancement Mode PHEMT (QE-PHEMT) unit cell is compared for wireless telephone $P A$ applications. While the HBT has advantages of smaller chip size and single supply with no drain switch operation, the QE-PHEMT has higher efficiency and better low voltage characteristics. PA power control and device design trade-offs for both technologies are also discussed.
\end{abstract}

\section{INTRODUCTION}

Both HBT and QE-PHEMT are attractive technologies for realizing high performance single supply power amplifiers for cellular telephone applications. Alpha Industries' QE-PHEMT and HBT devices are compared in this study. Their main features and device characteristics are described in Table 1. The quasi-E-mode definition is being applied to the PHEMT device because Idss $=10$ $\mathrm{uA} / \mathrm{mm}$, as compared to a $\mathrm{nA} / \mathrm{mm}$ range for true Emode devices. In the QE-PHEMT case, single supply operation is possible, but a drain switch is still required to shut the PA current off completely.

Table 1. Summary of the process features and main device characteristics of the QE-PHEMT and HBT processes studied.

\begin{tabular}{|l|l|}
\hline QE-PHEMT & HBT \\
\hline Selective dry etch & C-doped MOCVD epi \\
\hline Idss $=10 \mathrm{uA} / \mathrm{mm}$ & InGaP ledge \\
\hline $\mathrm{Vp}=0.3 \mathrm{~V}$ & \\
\hline $\mathrm{Imax}=380 \mathrm{~mA} / \mathrm{mm}$ & Beta $=100$ \\
\hline $\mathrm{Gm}=500 \mathrm{mS} / \mathrm{mm}$ & Ft $/ \mathrm{Fmax}=30 / 60 \mathrm{GHz}$ \\
\hline $\mathrm{Vbd}=15 \mathrm{~V}$ & $\mathrm{BVceo}=18 \mathrm{~V}$ \\
\hline $220 \mathrm{~mW} / \mathrm{mm} \mathrm{PAE}=55 \%$ & $0.8 \mathrm{~mW} / \mathrm{um}^{\wedge} 2 \mathrm{PAE}=58 \%$ \\
\hline $150 \mathrm{~mW} / \mathrm{mm} \mathrm{PAE}=76 \%$ & $0.6 \mathrm{~mW} / \mathrm{um} / 2 \mathrm{PAE}=67 \%$ \\
\hline$(@ 3.2 \mathrm{~V}, 900 \mathrm{MHz})$ & $($ @ $3.2 \mathrm{~V}, 900 \mathrm{MHz})$ \\
\hline
\end{tabular}

In an attempt to perform a fair comparison between these different device technologies, the unit QE-PHEMT and HBT cells were selected to achieve similar output power levels when operated at 900 $\mathrm{MHz}$ and a supply voltage of $3.2 \mathrm{~V}$. The power comparison is presented in Figure 1. The HBT cell occupied approximately $10-15 \%$ smaller total layout area than the QE-PHEMT cell. All of the measurements were performed on-wafer using an automated ATN load-pull stand with harmonic termination control.

Figure 2 shows, that when the supply voltage is decreased, the PAE of a QE-PHEMT remains above $70 \%$ even at $1 \mathrm{~V}$, whereas the PAE of the HBT decreases to slightly over $50 \%$. This occurs due to the higher knee voltage for the HBT resulting from the built-in voltage and from series ballast and stabilization resistors.

\section{DESIGN TRADE-OFFS}

In general, different sets of design trade-offs are observed for the HBT and the QE-PHEMT, as summarized in Table 2. In the case of the HBT, peak performance has to be de-rated to ensure a thermally robust, reliable, and VSWR mismatch stable device. For the QE-PHEMT, the primary challenges are to maintain good Po/PAE and Vbd/Ileak simultaneously and ensure repeatability of the process. Typical HBT failures are thermally activated and are related to the low impedance mismatch with its high current and high effective heat dissipation. On the other hand, QE-PHEMT failures are typically electrically activated and are related to the high impedance mismatch with its high drain-gate voltage excursions.

Table 3 summarizes the main variables affecting these design trade-offs.

Table 2. Summary of the QE-PHEMT and HBT trade-offs, which have to be balanced during the design process.

\begin{tabular}{|l|l|}
\hline QE-PHEMT & HBT \\
\hline Electrical-process stability & Electrical-thermal design \\
\hline Po/PAE - leakage & Po/PAE - reliability \\
\hline Po/PAE - breakdown & Po/PAE - VSWR stability \\
\hline
\end{tabular}

Table 3. The main set of variables for making good QE-PHEMT and HBT power amplifiers.

\begin{tabular}{|l|l|}
\hline QE-PHEMT & HBT \\
\hline Gate process & Stability/ballast resistors \\
\hline Etch uniformity & Transistor size \\
\hline Passivation/surface traps & Cell/PA layout \\
\hline Epi design & Epi design \\
\hline Repeatability & Bias/match point \\
\hline
\end{tabular}




\section{LOAD-PULL CHARACTERISTICS}

Figures 3 and 4 compare the $3.2 \mathrm{~V}, 900$ $\mathrm{MHz} \mathrm{HBT}$ and QE-PHEMT performance at various quiescent current levels. Gain compression (Fig. 4), PAE dynamics (Fig 3), as well as the dynamic current behavior under RF drive (Fig. 5 and 6) is quite similar for these devices, with exception of the $\mathrm{Vgs}=0$ and $\mathrm{Vbe}=0$ characteristics. Generally, the HBT starts with higher small-signal gain, but it compresses earlier, resulting in the lower peak PAE. This occurs due to the knee voltage limitation, as described before. Both devices are relatively insensitive to the quiescent bias with respect to the peak Pout and PAE values. However, QE-PHEMT is difficult to turn off with just $\mathrm{Vgs}=0$, as it starts to self-bias and turns on under RF drive much earlier than the HBT. Therefore, a VVA is typically required as part of the PA circuit to shut the QE-PHEMT stages completely off. On the other hand, in the HBT, this is not necessary, and adequate power control is normally achieved just by the Vbe variation.

Despite some fundamental technological differences between the HBT and the QE-PHEMT, some of the performance features of these devices look very similar, e.g. drain and collector current dynamics under RF drive, as indicated in Fig. 5 and 6. Another feature is inverse Class $F$ operation, preferred by both types of devices to achieve high efficiency. Figures 7 and 8 show the $2^{\text {nd }}$ harmonic tuning contours for the PAE of the QE-PHEMT and the HBT, respectively, measured at $900 \mathrm{MHz}$ and 3.2 $\mathrm{V}$. In both cases the fundamental load impedance was kept the same. It can be seen that the $2^{\text {nd }}$ harmonic open circuit results in peak PAE, whereas moving it to a short circuit results in about $20 \%$ drop in PAE. Peak efficiency is however larger for the QE-PHEMT which is consistent with the results presented in Figure 2.

\section{CONCLUSIONS}

In conclusion, HBT devices offer true single supply operation without a drain switch, occupy less space, and don't require a VVA for shutting the PA completely off in the off state. QE-PHEMTs offer approximately $10 \%$ higher power-added efficiency for the same level of output power (as shown in Fig. 9) and their performance advantage over HBTs increases as the supply voltage is dropped. QEPHEMT technology is also suitable for integrating PA's with low-noise amplifiers, transmit-receive switches, and attenuators on one chip for a complete $\mathrm{RF}$ front-end solution. In addition to lower PAE, the disadvantages of HBT include the requirement to trade-off peak performance to ensure reliability and stability with respect to the VSWR mismatch. Those for the QE-PHEMT include 10-15\% larger chip size, the VVA, and the drain switch requirements.

\section{DISCUSSION}

Presently, most of the next generation PA's are being designed in module form. In addition to many passive components, these $50 \Omega$ in-out modules contain other functional blocks, such as Si controller chip, diode detector, attenuator, switch, etc. In the end, performance and cost of the whole module and not an individual PA chip is what counts. This makes such issues as an additional VVA in front of the PA and drain switch less critical, since they can be incorporated as part of the overall module solution. Moreover, drain switch incorporation may be desirable to realize dynamic supply modulation, which is becoming a popular scheme for efficiency improvement at backed-off power conditions, and a VVA in front of the PA can be used to produce the desirable power control characteristic. These factors make the technology choice between the HBT and the QE-PHEMT for future PAs less obvious. Future battery trends and public acceptance of high-end (high efficiency) telephones will also affect the importance of the low voltage and high efficiency performance for the PA technology.

\section{REFERENCES}

[1] E. Glass et al, "A true enhancement-mode device technology for dual mode dual band power amplifier applications," in IEEE RFIC Symp Dig, pp. 135-138, 1999.

[2] S. Yoshida, Y. Wakabayashi, M. Kohno and K. Uemura, "Greater than 70\% PAE enhancement-mode GaAs HJFET power amplifier MMIC with extremely low leakage current," in IEEE IMS Dig, pp. 1183-1186, 1999.

[3] M. Nakamura, S. Wada, M. Abe, H. Kawasaki and I. Hase, "A buried p-gate HJFET for a power amplifier of digital wireless communication systems," in IEEE IMS Dig, pp. 10951098,1999

[4] N. Hara et al, "Current path optimized structure for high drain current density and high turn-on voltage E-mode HFETs," in IEEE GaAs IC Symp. Dig., 1998, pp. 198-201.

[5] D. Wu et al, "A 2W 65\% PAE single-supply enhancement mode power PHEMT for 3V PCS applications," in IEEE MTT Symp. Dig. pp. 1319-1322, 1997.

[6] Y. Tkachenko, A. Klimashov, Y. Zhao, C. Wei and D. Bartle "E-PHEMT for single-supply high efficiency power amplifiers, " in European Microwave Conf. Proceedings, 1999.

[7] C. Wei, Y. Tkachenko and D. Bartle "A new model for Emode power PHEMT and its optimum loading operation," in International Microwave and Optoelectronics Conference Proceedings, Rio de Janeiro, 1999.

[8] W. Abey, T. Moriuchi, R. Hajji, T. Nakamura, Y. Nonaka, E. Mitani, W. Kennan, and H. Dang, "A single supply high performance PA MMIC for GSM handsets using quasienhancement mode PHEMT," in IEEE International Microwave Symp. Dig. pp. 923-926, 2001. 


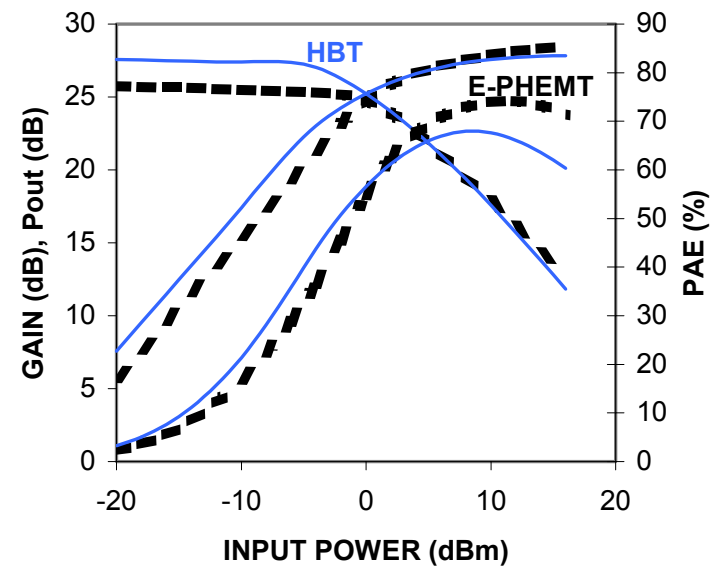

Figure 1. Unit cell performance of (-) HBT and (---) QEPHEMT at $900 \mathrm{MHz}$ and $3.2 \mathrm{~V}$.

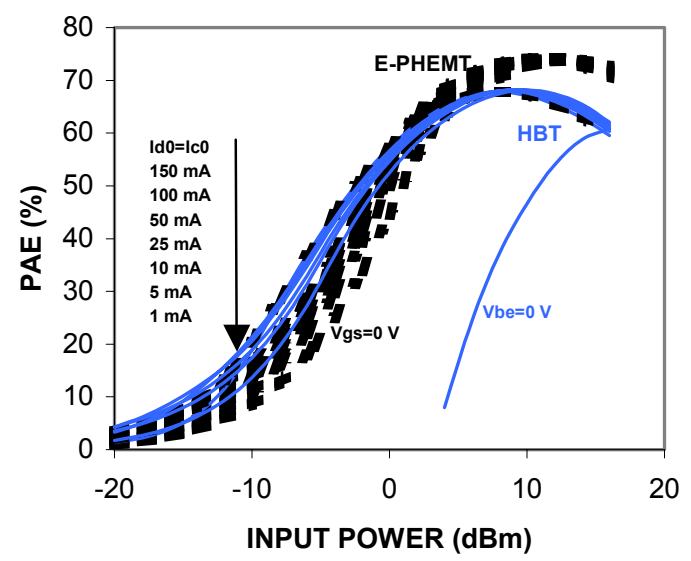

Figure 3. Power-added efficiency of (-) HBT and (---) QEPHEMT unit cells at $3.2 \mathrm{~V}$ and different bias current levels: 150 $100,50,25,10,5,1 \mathrm{~mA}$ and $\mathrm{Vgs}=\mathrm{Vbc}=0 \mathrm{~V}$.

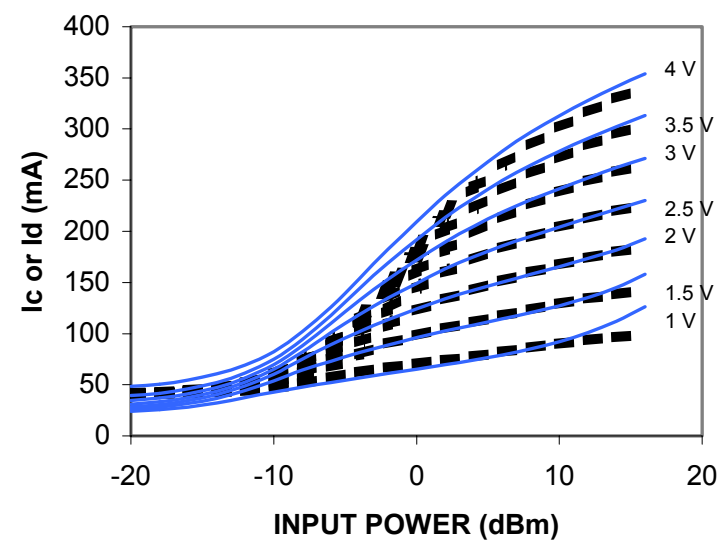

Figure 5. Collector and drain current comparison of (-) HBT and (---) QE-PHEMT unit cells at different supply voltages: 4, 3.5, 3 ... V.

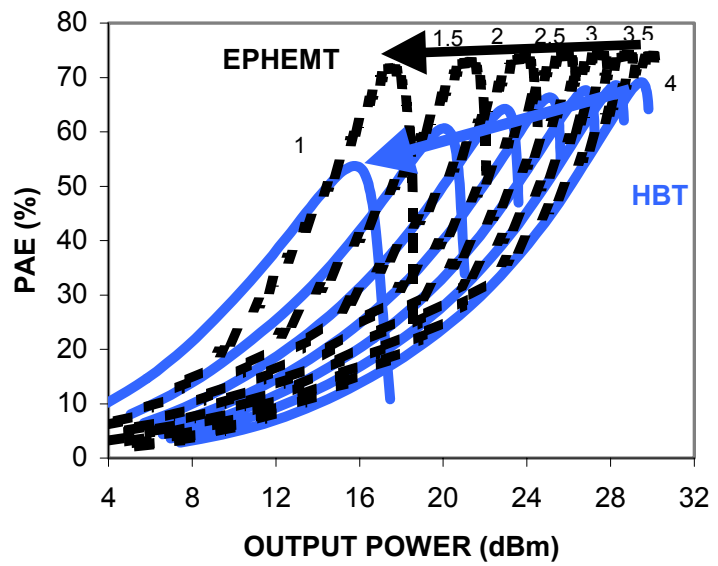

Figure 2. Power performance comparison of (-) HBT and (---) QE-PHEMT unit cell $\mathrm{s}$ at different supply voltages: $4,3.5 \ldots 1 \mathrm{~V}$.

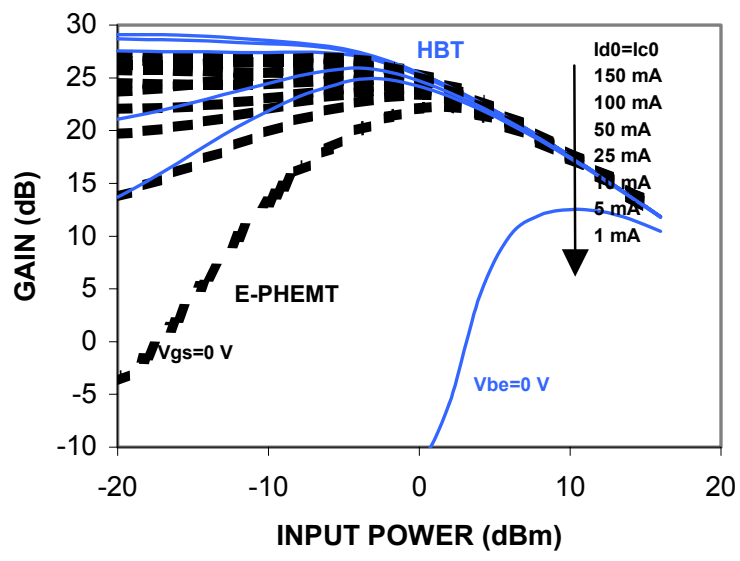

Figure 4. Gain of (-) HBT and (---) QE-PHEMT unit cells at 3.2 $\mathrm{V}$ and different bias current levels: $150,100,50,25,10,5,1 \mathrm{~mA}$ and $\mathrm{Vgs}=\mathrm{Vbc}=0 \mathrm{~V}$.

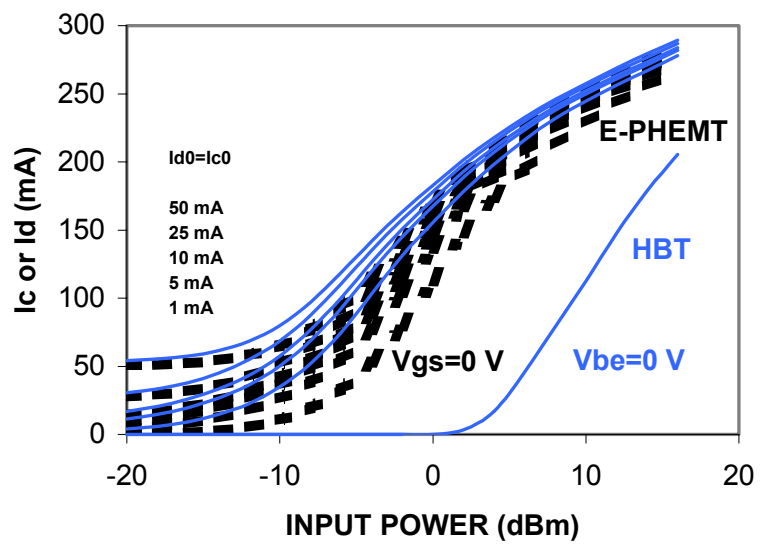

Figure 6. Collector and drain current of (-) HBT and (---) QEPHEMT unit cells at $3.2 \mathrm{~V}$ and different bias current levels: 150 , $100,50,25,10,5,1 \mathrm{~mA}$ and $\mathrm{Vgs}=\mathrm{Vbc}=0 \mathrm{~V}$. 


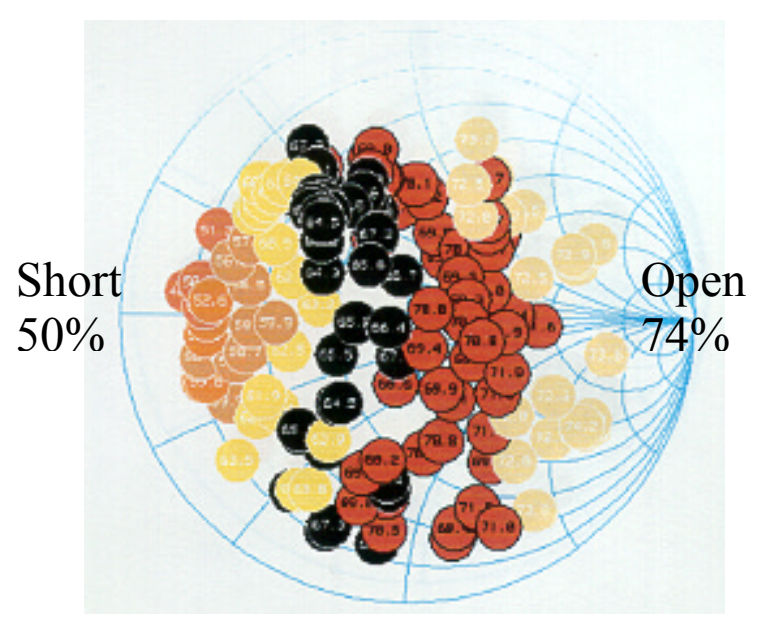

Figure 7. Measured QE-PHEMT PAE vs. $2^{\text {nd }}$ harmonic load at fo $=$ $900 \mathrm{MHz}$ and $\mathrm{Vd}=3.2 \mathrm{~V}$.

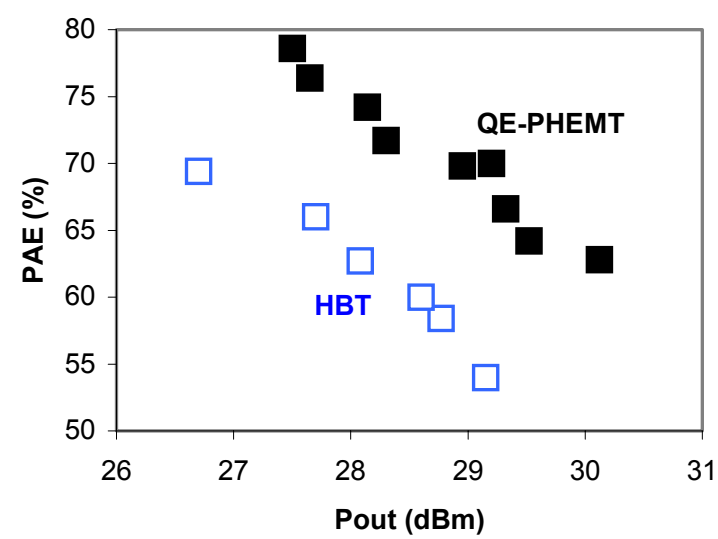

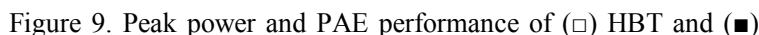
QE-PHEMT unit cells at $900 \mathrm{MHz}, 3.2 \mathrm{~V}$ and $40 \mathrm{~mA}$. Each point represents a different matching condition varying from max Pout to max PAE tuning and including the Po-PAE trade-off states inbetween.

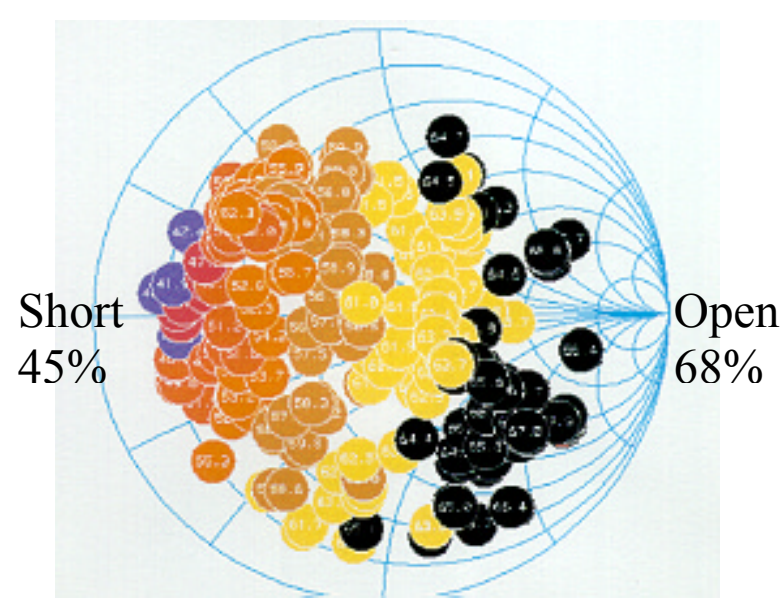

Figure 8. Measured HBT PAE vs. $2^{\text {nd }}$ harmonic load at fo $=900$ $\mathrm{MHz}$ and $\mathrm{Vc}=3.2 \mathrm{~V}$. 